\title{
A Hug Behavior Generation Model Based on Analyses of Human Behaviors for Hug Robot System
}

\author{
Mitsuru Jindai ${ }^{1, a}$, Shunsuke Ota ${ }^{1}$ and Tohru Sasaki ${ }^{1}$ \\ ${ }^{1}$ Graduate School of Science and Engineering, University of Toyama, 3190 Gofuku, Toyama, 930-8555, Japan
}

\begin{abstract}
In human face-to-face communication, embodied sharing using the synchrony of embodied rhythms is promoted by embodied interactions. In particular, embodied interactions with direct contact are considered to synchronize embodied rhythms effectively. Hug behavior is one of the types of embodied interactions that involve direct contact. In hug behaviors, humans whole-body contact with each other. In the case of interaction between a human and a robot, the robot synchronizes embodied rhythms effectively using hug behaviors. Thus, a behavior model that can generate a hug behavior with humans is required for robots. Therefore, in this paper, we analyzed hug behaviors between humans and proposed a hug behavior generation model for a hug robot system based in the results of the analysis. This model generates a hug behavior in response to human behavior when a human requests a hug from the robot. Next, we developed a hug robot system that uses the proposed hug behavior generation model. Using the robot system, we demonstrated the effectiveness of the proposed hug behavior generation model by means of a sensory evaluation.
\end{abstract}

\section{Introduction}

In human face-to-face communication, embodied sharing using the synchrony of embodied rhythms is promoted by embodied interactions. Therefore, embodied interactions are important to the smooth initiation of coexistence and communication. In the case of interaction between a human and a robot, the robot can begin to communicate and coexist smoothly with the human without producing feelings of aversion in the human if the robot generates an embodied interaction that is emotionally acceptable to the human. A robot that can generate such an interaction is emotionally acceptable to humans because it produces feelings of security. Embodied interactions that involve direct contact are considered to synchronize embodied rhythms particularly effectively.

In hug behaviors, humans make whole-body contact with each other. Therefore, hug behaviors are expected to synchronize embodied rhythms effectively. In the case of an interaction between a human and a robot, the robot can effectively synchronizes embodied rhythms by hug behaviors. Hug behaviors between robots and humans have been examined in previous studies [1]. These studies examined remote hug communications with partners or hugging robots. However, no previous studies have examined the hand behaviors of hugs or the timing of their initiation. Thus, a behavior model that can generate hug behavior with humans is required.

\footnotetext{
${ }^{\text {a }}$ Corresponding author : jindai@eng.u-toyama.ac.jp
} 
Therefore, in this paper, we propose a hug behavior generation model based on an analysis of human behaviors. We first analyze hug behaviors between humans. Based on the results of this analysis, we propose a hug behavior generation model for a hug robot system. This model generates a hug behavior in response to human behavior when a human requests a hug from the robot. Furthermore, a hug robot system that uses the proposed hug behavior generation model is developed. Using the developed robot system, the effectiveness of the proposed hug behavior generation model is demonstrated by sensory evaluation.

\section{Analysis of Hug Behavior between Humans}

\subsection{Hug Experiment}

Hug behaviors between humans were measured experimentally to provide a basis for modelling similar behaviors between a human and a robot. In the experiment, human behaviors were measured using a three-dimensional motion capture system (VICON). The request side and response side were determined beforehand. Eleven reflection markers (head, both shoulders, both elbows, both wrists, both hands, and both ankles) were attached to the request-side and response-side subjects. Furthermore, microphones were used to input voice utterances of the request-side and response-side subjects.

Figure 1 shows a typical experimental scene. The subjects initially stand face to face at a distance of 3,000 $\mathrm{mm}$, with their arms extended downward. The request-side subject approaches the responseside subject and starts to move his or her hands at an arbitrary time to hug the response-side subject. The response-side subject then moves his or her hands in response to this behavior by the request-side subject. The subjects were 30 healthy right-handed students (15 pairs) aged between 20 and 24, and each pair hugged 10 times.

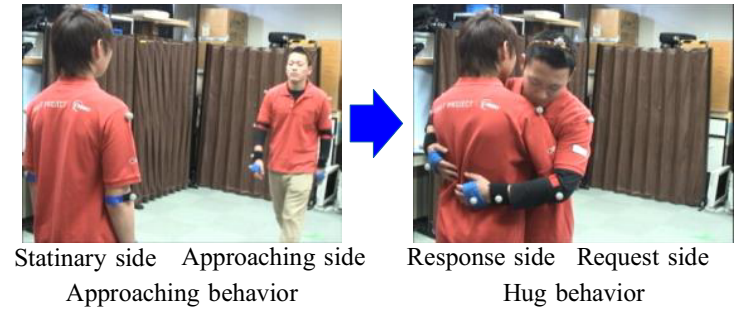

Figure 1. Experimental scene of hug behavior between humans

\subsection{Analysis Results}

The rotational behaviors of the subjects' arm joints during a hug were analyzed to determine the hand trajectories. Furthermore, the timing of the initiation of hand behavior was analyzed.

\subsubsection{Angular velocities of joints}

The angular velocities of the joints of the response-side subjects were measured to analyze the hand behaviors of the response-sides subjects during hug behaviors between humans. Examples of measured velocity profiles are shown in Figures 2 and 3. Figure 2 shows angular velocity profiles of the elbow and shoulder, and Figure 3 shows an angular velocity profile of the external and internal rotation of the upper arm. Based on these figures, the velocity profiles were classified as belonging to either of two types. The first type is a bell-shaped velocity profile, as shown in Figure 2. In this velocity profile, there is a positive or a negative peak. The second type is a wave-shaped velocity profile, as shown in Figure 3. In this velocity profile, there is both a positive and a negative peak. The velocity profiles of the shoulder, elbow, forearm, and wrist were classified as being bell-shaped. The 
velocity profile of the external and internal rotation of the upper arm was classified as being waveshaped. These figures illustrate that the angular velocity profiles have smooth transitions. This indicates that the response-side subjects moved their right and left hands along smooth trajectories.

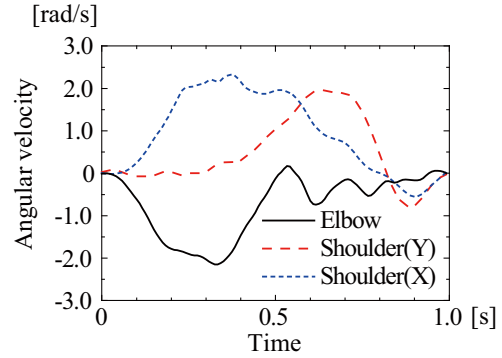

Figure 2. Velocity profiles of elbow and shoulder

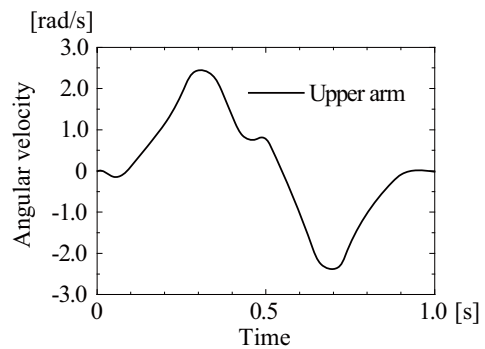

Figure 3. Velocity profiles of upper arm

\subsubsection{Initiation of hand behaviors}

In hug behaviors between humans, the response-side humans move their hands according to the behaviors of the request-side humans. Therefore, the timing of the initiation of hand behaviors is important for smooth hug behaviors. In this study, the time gaps between hand behaviors of the response-side subjects and various behaviors of the request-side subjects were measured. Hand behaviors, utterances of voice greetings, and the termination of approaching were the focuses of the observed hug behavior of the request-side subjects.

(1) Time gap of initiation of hand behaviors

The time gaps between the initiation of hand behaviors by the response side and the request side during the hug behaviors were measured. Figure 4 shows a histogram of the time gaps. As this figure shows, the time gaps that were measured for most of the subjects were widely distributed between -0.1 $\mathrm{s}$ and $1.2 \mathrm{~s}$, with a peak of $0.3 \mathrm{~s}$. A negative time gap indicates that the hand behavior of the response side began before the hand behavior of the request side, whereas a positive time gap indicates that the hand behavior of the response side began after the initiation of the hand behavior of the request side. These results suggest that most of the response-side subjects began their hug behavior $0.3 \mathrm{~s}$ after the initiation of the hug behavior of the request-side subjects.
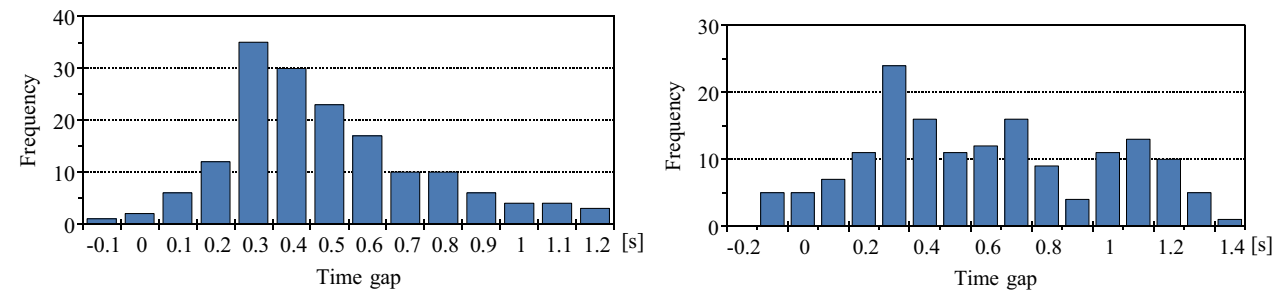

Figure 4. Time gap of hand behaviors

Figure 5. Time gap between hand behavior and voice

(2) Time gap between initiation of hand behavior and voice utterance

The time gaps between the initiation of hand behaviors of the response-side subjects and the initiation of voice utterances of the request-side subjects during the hug behaviors were measured. Figure 5 shows a histogram of the time gaps. As the figure shows, the time gaps measured for most of the subjects were widely distributed between $-0.1 \mathrm{~s}$ and $1.4 \mathrm{~s}$, with a peak of $0.3 \mathrm{~s}$. A positive time gap indicates that the hand behavior began after the voice greeting. These results suggest that most of the response-side subjects begin their hug response behavior $0.3 \mathrm{~s}$ after the voice utterance of the requestside subjects.

(3) Time gap between initiation of hand behavior and termination of approaching

The time gaps between the initiation of hand behaviors of the response-side subjects and the termination of approaching of the request-side subjects were also measured. Figure 6 shows a histogram of the time gaps. As this figure shows, the time gap measured for most of the subjects were 
widely distributed between $-0.4 \mathrm{~s}$ and $1.2 \mathrm{~s}$, with a peak at $0.4 \mathrm{~s}$. A positive time gap indicates that the hand behavior began after the termination of approaching. These results suggest that most of the response-side subjects began their hug response behavior $0.4 \mathrm{~s}$ after the termination of approaching behavior by the request-side subject.

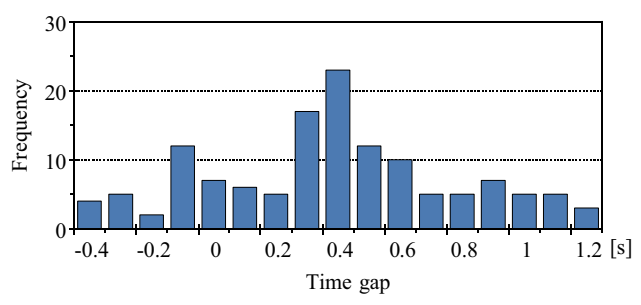

Figure 6. Time gap between hand behavior and termination

\section{Hug Behavior Generation Model}

A hug behavior generation model is proposed for generation of hug behavior. Based on the analysis of the hug behaviors between humans, it was determined that the velocity patterns of human arm joint movements during hug behavior are bell-shaped and wave-shaped. Therefore, a hug behavior generation model is proposed that can generate hug behavior described by these two velocity patterns.

\subsection{Bell-Shaped Velocity Pattern}

A bell-shaped velocity pattern with an adjustable peak time is generated on the basis of the minimum jerk model [2]. The minimum jerk model can accurately reproduce the point-to-point motion of human hands. In this model, the acceleration and velocity patterns exhibit smooth transitions. Behaviors that are generated on the basis of the minimum jerk model are expected to be preferred by humans. Therefore, a bell-shaped velocity pattern is generated from two velocity patterns that are generated by the minimum jerk model [3].

\subsection{Wave-Shaped Velocity Pattern}

The wave-shaped velocity pattern is generated using a fifth-order curve. The wave-shaped velocity pattern is shown in Figure 7. In the wave-shaped velocity pattern, its shape is determined by a movement time, maximum velocity, and initial and target angles. The fifth-order curve is given by Equation (1). The movement time and peak time are denoted by $T_{f}$ and $T_{p}$, respectively. The time $T_{c}$ is the time at which the velocity becomes 0 . The value of the constant $K$ is calculated from the initial angle $R_{0}$ and the target angle $R_{f}$, using Equation (2). The peak time $T_{p}$ is calculated using Equation (3). Using these equations, the time $T_{c}$ is determined as the velocity at time $T_{p}$ reaches the maximum velocity $V_{\max }$.

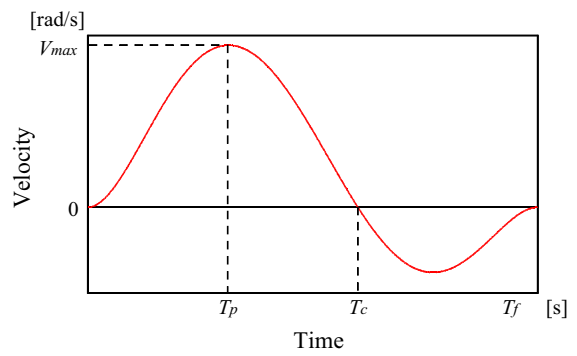

$$
\begin{aligned}
& V(t)=K\left(T_{f}-t\right)^{2}\left(T_{c}-t\right) t^{2} \\
& K=\frac{60\left(R_{f}-R_{0}\right)}{2 T_{f}^{5} T_{c}-T_{f}{ }^{6}} \\
& T_{p}=\frac{\left(4 T_{c}+3 T_{f}\right)-\sqrt{16 T_{c}{ }^{2}-16 T_{f} T_{c}+9 T_{f}{ }^{2}}}{10}
\end{aligned}
$$

Figure 7. Velocity profiles of elbow and shoulder 


\subsection{Generated Hug behavior}

In the proposed hug behavior generation model, the rotational behaviors of the shoulder, elbow, forearm, and wrist joints are generated using the bell-shaped velocity pattern, and the rotational behavior of the upper arm is generated using the wave-shaped velocity pattern. To produce a hand behavior similar to that of a human, the movement time, maximum velocity, peak time, and initial and target angles are matched to those of humans, as determined from the analysis of hug behaviors between humans.

\section{Experiment Using Hug Robot System}

A hug robot system that uses the proposed hug behavior generation model was developed as shown in figure 8. Using the hug robot system, an experiment was performed. Figure 9 shows a typical experimental scene. Three modes were used in this experiment. In mode (a), the robot began hug behavior $0.3 \mathrm{~s}$ after the initiation of hug behavior by a human. In mode (b), the robot began hug behavior $0.3 \mathrm{~s}$ after a voice utterance by a human. In mode (c), the robot began hug behavior $0.4 \mathrm{~s}$ after the termination of approaching behavior by a human. These modes were selected based on the results of the analysis of hug behaviors between humans, as discussed in the previous section.

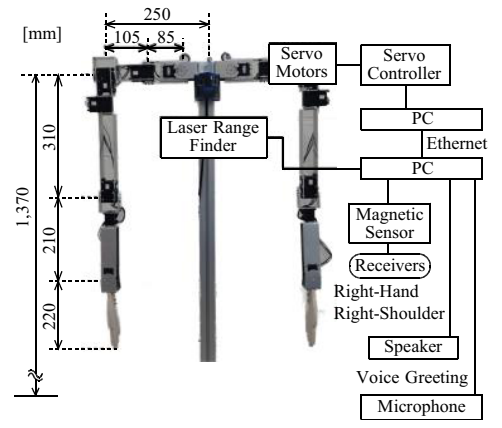

Figure 8. Hug Robot System

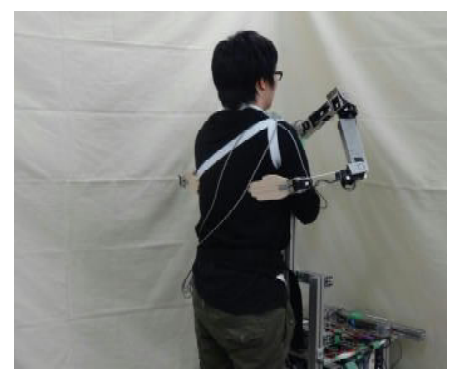

Figure 9. Experimental scene

\subsection{Experimental Method}

In the experiment, a human and the robot initially stood face to face at a distance of 3,000 mm. Next, the human approached the robot and started to move his or her hand at an arbitrary time to request a hug. The robot then moved its hand in response to this behavior. The experiment was performed for a hug behavior accompanied by a voice greeting. The robot uttered a voice greeting $0.1 \mathrm{~s}$ after the initiation of its hand behavior. This timing was selected based on the analysis of hug behaviors between humans.

In the sensory evaluation, paired comparison was made first. Subsequently, seven-point bipolar rating was made. Paired comparison was performed for all six pairs of mode combinations, and then seven-point bipolar rating on a scale from -3 (not at all) to 3 (extremely) for the following four items: "Ease of hug," "Velocity (Comfortable)," "Security," and "Politeness." The subjects used the modes randomly. The subjects were 30 healthy students aged between 20 and 24 years. All subjects were males 


\subsection{Experimental results}

The result of the paired comparison is shown in Table 1. The number of subjects that preferred the column mode to the row mode is shown in each cell of the table. Table 1 indicates that mode (b) was preferred by the largest number of subjects.

The results of the seven-point bipolar rating are shown in Figure 10. Modes (b) and (c) were rated higher than mode (a), with significant differences at the 1 and $5 \%$ significance levels for the "Politeness" item. However, there were no significant differences between modes (b) and (c) for any of the items.

The results obtained using the paired comparison indicate that mode (b) was the preferred mode, and using the seven-point bipolar rating scale, all items related to mode (b) had positive values. Therefore, it can be concluded that the hug behavior described by mode (b) is preferred by humans over that described by the other modes.

Table 1. Result of the paired comparison

\begin{tabular}{|l|r|r|r|r|}
\hline & (a) & (b) & (c) & \multicolumn{1}{|c|}{ Total } \\
\hline (a) & & 24 & 34 & 58 \\
\hline (b) & 36 & & 36 & 72 \\
\hline (c) & 26 & 24 & & 50 \\
\hline
\end{tabular}
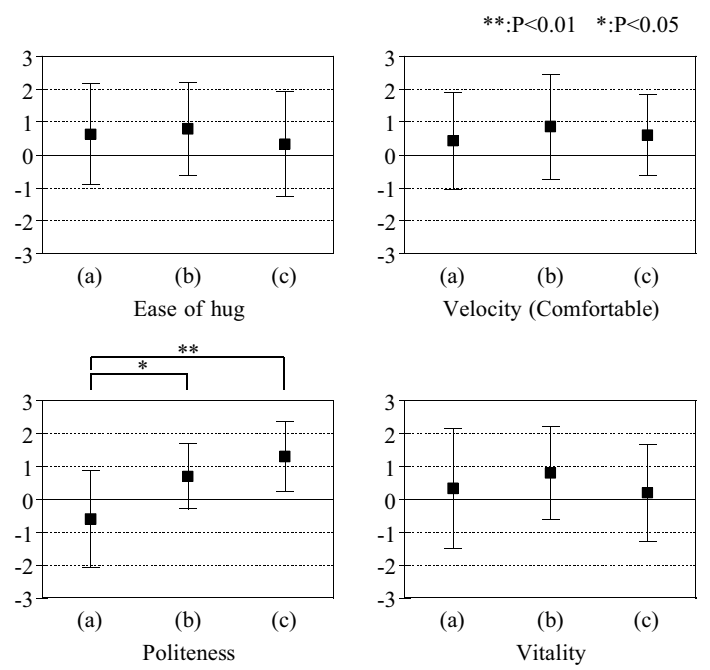

Figure 10. Results of seven-point bipolar rating

\subsection{Discussion of Experimental Results}

The experimental results suggest that in a hug interaction between a human and a robot, the robot should move its hands in response to a voice utterance by the human when the robot is the responder. The timing of a voice utterance by a human was found to be important to the generation of smooth hug behavior by a robot. Furthermore, a hug robot system can generate a hug behavior that is preferred by many humans. Therefore, the effectiveness of the proposed model has been demonstrated.

\section{Conclusion}

In this paper, we proposed a hug behavior generation model based on an analysis of human hug behaviors. This model generates a hug behavior in response to human behavior when a human requests a hug from a robot. Furthermore, a hug robot system that uses the proposed hug behavior generation model was developed. From experiment, in a hug interaction between a human and a robot, 
the timing of a voice utterance by the human was found to be important to the generation of smooth hug behavior by the robot. Furthermore, the effectiveness of the proposed hug behavior generation model has been demonstrated.

\section{Acknowledgment}

This work was supported by KAKENHI Grant Number 25330239 of the Japan Society for the Promotion of Science (JSPS), Japan.

\section{References}

1. J. Nakanishi, K. Kuwamura, T. Minato, S. Nishio and H. Ishiguro, Evoking Affection for a Communication Partner by a Robotic Communication Medium, Proceedings of the 1st International Conference on Human-Agent Interaction, III-1-4 (2013)

2. T.Flash and N.Hogan, The Coordination of Arm Movements: An Experimentally Confirmed Mathematical Model, Journal of Neurosciences Vol.5, No.7, pp.1688-1703 (1985)

3. M. Jindai and T. Watanabe, Development of a Handshake Request Motion Model Based on Analysis of Handshake Motion between Humans, Proceedings of the 2011 IEEE/ASME International Conference on Advanced Intelligent Mechatronics, pp.560-565 (2011) 